\title{
Abstract Gringo
}

\author{
MARTIN GEBSER $*$ \\ Aalto University, HIIT, Finland \\ University of Potsdam, Germany \\ gebser@cs.uni-potsdam.de
}

AMELIA HARRISON $\dagger$

Univeristy of Texas at Austin, USA

ameliaj@cs.utexas.edu

\section{ROLAND KAMINSKI*}

University of Potsdam, Germany

kaminski@cs.uni-potsdam.de

VLADIMIR LIFSCHITZ $\dagger$

Univeristy of Texas at Austin, USA

vl@cs.utexas.edu

\section{TORSTEN SCHAUB $* \ddagger$}

University of Potsdam, Germany INRIA Rennes, France

torsten@cs.uni-potsdam.de

submitted 1 January 2003; revised 1 January 2003; accepted 1 January 2003

\begin{abstract}
This paper defines the syntax and semantics of the input language of the ASP grounder GRINGO. The definition covers several constructs that were not discussed in earlier work on the semantics of that language, including intervals, pools, division of integers, aggregates with non-numeric values, and lparse-style aggregate expressions. The definition is abstract in the sense that it disregards some details related to representing programs by strings of ASCII characters. It serves as a specification for GRINGO from Version 4.5 on.
\end{abstract}

* Supported by AoF (grant 251170) and DFG (grants SCHA 550/8 and 550/9).

$\dagger$ Partially supported by the National Science Foundation under Grant IIS-1422455.

$\ddagger$ Affiliated with Simon Fraser University, Canada, and IIIS Griffith University, Australia. 


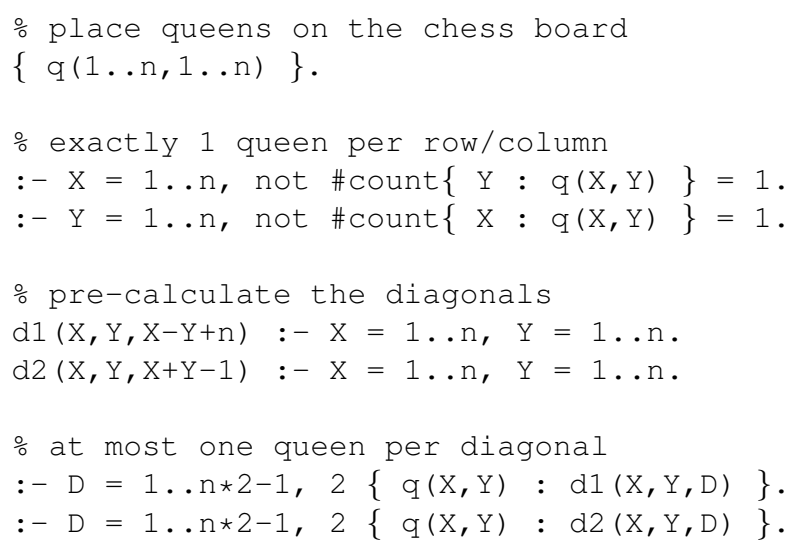

Table 1. An ASP solution to the n-queens problem.

\section{Introduction}

Version 4.0 of the ASP grounder GRINGO was released in March of 2013. ${ }^{1}$ Harrison et al. (2014) defined the semantics of a subset of its input language in terms of stable models of infinitary propositional formulas (Truszczynski 2012).

That subset does not include, however, several constructs that are frequently used in ASP programs. One such construct is integer intervals. Take, for instance, the ASP solution to the $n$-queens problem shown in Table 1. (It is similar to one of the solutions in the language of Version 3 presented by Gebser et al. (2011).) Intervals are used in each rule of this program. To include intervals, we have to modify the semantics from Harrison et al. (2014) in two ways. First, we have to say that an arithmetic term denotes, generally, a finite set of integers, not a single integer. (And it is not necessarily a set of consecutive integers, because the language of GRINGO allows us to write $(1 \ldots 3) * 2$, for instance. This expression denotes the set $\{2,4,6\}$.) Second, in the presence of intervals we cannot treat a choice rule $\{A\}$ as shorthand for the disjunctive rule $A$; not $A$ as proposed by Ferraris and Lifschitz (2005). Indeed, the first rule of the program in Table 1 has $2^{n^{2}}$ stable models; the rule

$$
\mathrm{q}(1 \ldots \mathrm{n}, 1 \ldots \mathrm{n}) ; \operatorname{not} \mathrm{q}(1 \ldots \mathrm{n}, 1 \ldots \mathrm{n})
$$

has only 2 stable models.

Another feature of GRINGO not covered by Harrison et al. (2014), which is somewhat similar to integer intervals, is pooling. Pooling is used, for instance, in the head of the rule

$$
\mathrm{p}(\mathrm{X} ; \mathrm{Y}):-\mathrm{q}(\mathrm{X}, \mathrm{Y}) \text {. }
$$

(Note that a semicolon, not a comma, separates $X$ from $Y$ in the head.) This rule has the

${ }^{1}$ http://potassco.sourceforge.net/ 
same meaning as the pair of rules

$$
\begin{aligned}
& p(X):-q(X, Y) \\
& p(Y):-q(X, Y)
\end{aligned}
$$

Pooling is often used to abbreviate a set of facts. For instance, instead of

$$
\mathrm{p}(\mathrm{a}, 5) \cdot \mathrm{p}(\mathrm{b}, 10) \cdot \mathrm{p}(\mathrm{c}, 12)
$$

we can write $\mathrm{p}(\mathrm{a}, 5 ; \mathrm{b}, 10 ; \mathrm{c}, 12)$. In this paper, we talk about "pools"-groups of terms such as $a, 5 ; b, 10 ; \mathrm{c}, 12$.

Yet another limitation of the proposal from Harrison et al. (2014) is related to the difference between "dlv-style" aggregates, such as

$$
\text { not } \# \operatorname{count}\{\mathrm{Y}: \mathrm{q}(\mathrm{X}, \mathrm{Y})\}=1
$$

in the second rule of the program shown in Table 1, and "lparse-style" aggregates, such as

$$
2\{\mathrm{q}(\mathrm{X}, \mathrm{Y}): \mathrm{d} 2(\mathrm{X}, \mathrm{Y}, \mathrm{D})\}
$$

in the last rule of the program. Both expressions have to do with counting. Syntactically, the difference is that in expression (1) both the name of the aggregate (\#count) and the binary relation applied to the result of counting and a constant $(=)$ are shown explicitly; in (2), the fact that the constant 2 occurs on the left, in the lower bound position, tells us that the relation $\leq$ is applied to that number and to the result of counting. More importantly, there is a difference between the kinds of objects that we count. In case of expression (1) we count, for a given value of $X$, the values of the variable $Y$ such that $q(X, Y)$ belongs to the stable model. In case of (2) we count, for a given value of $D$, the atoms $q(X, Y)$ that belong to the stable model and satisfy an additional condition: $\mathrm{d} 2(\mathrm{X}, \mathrm{Y}, \mathrm{D})$ belongs to the model as well. Thus the atom in front of the colon in (2) plays two roles: it tells us what to count, and it gives a condition on the stable model that needs to be checked.

The language studied by Harrison et al. (2014) does not include lparse-style aggregates. The easiest way to add such aggregates is to treat them as abbreviations. For instance, (2) can be viewed as shorthand for the dlv-style expression

$$
2<=\# \text { count }\{\mathrm{q}(\mathrm{X}, \mathrm{Y}): \mathrm{q}(\mathrm{X}, \mathrm{Y}), \mathrm{d} 2(\mathrm{X}, \mathrm{Y}, \mathrm{D})\} \text {. }
$$

(In this paper we adopt a more elaborate translation that allows us to accommodate negated atoms in front of the colon.) In this expression, the first occurrence of $q(X, Y)$ is syntactically a term, and the second is an atom. Thus treating (2) as an abbreviation depends on the possibility of using the same symbol as a function and as a predicate. This is customary in Prolog, but not in first-order logic, and this was not allowed by Harrison et al. (2014).

Our goal is to define the syntax and semantics of the language AG (short for Abstract Gringo) - a large subset of the input language of GRINGO that includes the features mentioned previously and a few other constructs not described by Harrison et al. (2014). This 
is similar to the work that has led to the definition of the ASP Core language (Calimeri et al. 2012). ${ }^{2,3}$

The semantics of AG defined in this paper serves a specification for GRINGO from Version $4.5 \mathrm{on}$. It can be used to prove the correctness of programs written in its input language. As an example, in the electronic appendix we prove the correctness of the program shown in Table 1.

AG is abstract in the sense that its definition disregards some details related to representing programs by strings of ASCII characters. For example, semicolons are used in the input language of GRINGO in at least three ways: to denote disjunction in the head of a rule, conjunction in the body, and pooling within an atom. In AG, three different symbols play these different roles. The richer alphabet of AG makes it easier to define the semantics of the language and to reason about ASP programs.

\section{Syntax of AG}

\subsection{Symbols and Terms}

We assume that five sets of symbols are selected: numerals, symbolic constants, negated constants, variables, and aggregate names. We assume that a 1-1 correspondence between the set of symbolic constants and the set of negated constants is chosen. For every symbolic constant $p$, the corresponding negated constant will be called its strong negation and denoted by $\tilde{p}$. Further, we assume that these sets do not contain the symbols

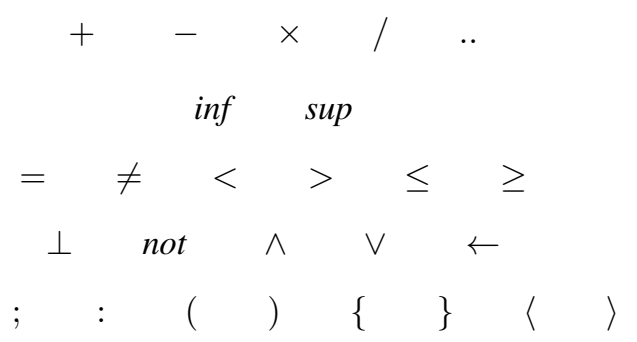

and that they are pairwise disjoint. All these symbols together form the alphabet of AG, and $\mathrm{AG}$ rules will be defined as strings over this alphabet.

When a symbol is represented in ASCII, its type is determined by its first two characters. For instance, a numeral starts with a digit or - followed by a digit. A symbolic constant starts with a lower-case letter. A negated constant starts with - followed by a lower-case letter. A variable starts with an upper-case letter, and an aggregate name starts with \#. (The

\footnotetext{
${ }^{2}$ Syntactically, AG is essentially an extension of ASP Core. But it does not include extra-logical constructs, such as weak constraints and queries. The semantics of aggregates in AG is based on the approach of Ferraris (2005) and thus is not equivalent to the semantics of aggregates in ASP Core when aggregates are used recursively in the presence of negation. Among the language constructs that are not in ASP Core, in AG we find pooling, intervals, and conditional literals. (These constructs originally appeared in the input language of LPARSE, but in AG they are more general; for instance, interval bounds may contain variables, and restrictions to "domain predicates" have disappeared.) Unlike ASP Core, AG supports aggregates in rule heads; see Section 3.

3 The definition of ASP Core does not refer to infinitary objects, such as infinitary propositional formulas used in this paper. But it appears that infinitary objects of some kind will be required to correct the oversight in (Calimeri et al. 2012, Section 2.2) - the set inst $\left(\left\{e_{1} ; \ldots ; e_{n}\right\}\right.$ ), included in the body of a rule in the process of instantiation, can be infinite.
} 


\begin{abstract}
Gringo
strings \#false, \#inf, and \#sup, which represent $\perp$, inf, and sup, also start with \#.) The symbols $\langle$ and $\rangle$ (which are used to indicate the boundaries of a tuple within a term) correspond to the ASCII characters ( and ). ${ }^{4}$ Each of the symbols (3)-(7) except for $\wedge$ and $\vee$ has a unique ASCII representation; the symbols $\wedge$ and $\vee$ can be represented by semicolons and in some cases also by commas.

We assume that a 1-1 correspondence between the set of numerals and the set $\mathbf{Z}$ of integers is chosen. For every integer $n$, the corresponding numeral will be denoted by $\bar{n}$.

Terms are defined recursively, as follows:

- all numerals, symbolic constants, and variables are terms;

- if $f$ is a symbolic constant and $\mathbf{t}$ is a tuple ${ }^{5}$ of terms then $f(\mathbf{t})$ is a term;

- if $t_{1}$ and $t_{2}$ are terms and $\star$ is one of the symbols (3) then $\left(t_{1} \star t_{2}\right)$ is a term;

- if $\mathbf{t}$ is a tuple of terms then $\langle\mathbf{t}\rangle$ is a term.
\end{abstract}

In a term of the form $f()$ the parentheses can be dropped, so that every symbolic constant can be viewed as a term. In a term of the form $\left(t_{1} \star t_{2}\right)$ we will drop the parentheses when it should not lead to confusion. A term of the form $(\overline{0}-t)$ can be abbreviated as $-t$.

A term, or a tuple of terms, is precomputed if it contains neither variables nor symbols (3). We assume a total order on precomputed terms such that inf is its least element, sup is its greatest element, and, for any integers $m$ and $n, \bar{m} \leq \bar{n}$ iff $m \leq n$.

We assume that for each aggregate name $\alpha$ a function $\widehat{\alpha}$ is chosen that maps every set of tuples of precomputed terms to a precomputed term. ${ }^{6}$ The AG counterparts of the aggregates implemented in Version 4.5 of GRINGO are defined below using the following terminology. If the first member of a tuple $\mathbf{t}$ of precomputed terms is a numeral $\bar{n}$ then we say that the integer $n$ is the weight of $\mathbf{t}$; if $\mathbf{t}$ is empty or its first member is not an integer then the weight of $\mathbf{t}$ is 0 . For any set $T$ of tuples of precomputed terms,

- $\widehat{\operatorname{count}}(T)$ is the numeral corresponding to the cardinality of $T$ if $T$ is finite, and sup otherwise;

- $\widehat{\operatorname{sum}}(T)$ is the numeral corresponding to the sum of the weights of all tuples in $T$ if $T$ contains finitely many tuples with non-zero weights, and 0 otherwise;

- $\widehat{\operatorname{sum}+}(T)$ is the numeral corresponding to the sum of the weights of all tuples in $T$ whose weights are positive if $T$ contains finitely many such tuples, and sup otherwise;

- $\widehat{\min }(T)$ is sup if $T$ is empty, the least element of the set consisting of the first elements of the tuples in $T$ if $T$ is a finite non-empty set, and inf if $T$ is infinite;

- $\widehat{\max }(T)$ is inf if $T$ is empty, the greatest element of the set consisting of the first elements of the tuples in $T$ if $T$ is a finite non-empty set, and sup if $T$ is infinite.

\footnotetext{
${ }^{4}$ When an AG term representing a tuple of length 1, such as $\langle a\rangle$, is represented in ASCII, a comma is appended to the tuple: $(a$,$) .$

${ }^{5}$ In this paper, when we refer to a tuple of syntactic objects, we mean that the tuple may be empty and that its members are separated by commas.

${ }^{6}$ This understanding of $\widehat{\alpha}$ is different from that given by Harrison et al. (2014, Section 3.3). There, $\widehat{\alpha}$ is understood as a function that maps tuples of precomputed terms to elements of $\mathbf{Z} \cup\{\infty,-\infty\}$.
} 
Gebser et al.

\subsection{Atoms, Literals, and Choice Expressions}

A pool is an expression of the form $\mathbf{t}_{1} ; \ldots ; \mathbf{t}_{n}$ where $n \geq 1$ and each $\mathbf{t}_{i}$ is a tuple of terms. ${ }^{7}$ In particular, every tuple of terms is a pool.

An atom is a string of one of the forms $p(P), \tilde{p}(P)$ where $p$ is a symbolic constant and $P$ is a pool. In an atom of the form $p()$ or $\tilde{p}()$ the parentheses can be dropped, so that all symbolic constants and all negated constants can be viewed as atoms.

For any atom $A$, the strings

$$
A \quad \operatorname{not} A \quad \operatorname{not} \text { not } A
$$

are symbolic literals. ${ }^{8}$ An arithmetic literal is a string of the form $t_{1} \prec t_{2}$ where $t_{1}, t_{2}$ are terms and $\prec$ is one of the symbols (5).

A conditional literal is a string of the form $H: \mathbf{L}$ where $H$ is a symbolic or arithmetic literal or the symbol $\perp$ and $\mathbf{L}$ is a tuple of symbolic or arithmetic literals. If $\mathbf{L}$ is empty then we will drop the colon, so that every symbolic or arithmetic literal can be viewed as a conditional literal. ${ }^{9}$

An aggregate atom is a string of one of the forms

$$
\begin{gathered}
\alpha\left\{\mathbf{t}_{1}: \mathbf{L}_{1} ; \ldots ; \mathbf{t}_{n}: \mathbf{L}_{n}\right\} \prec s \\
s \prec \alpha\left\{\mathbf{t}_{1}: \mathbf{L}_{1} ; \ldots ; \mathbf{t}_{n}: \mathbf{L}_{n}\right\} \\
s_{1} \prec_{1} \alpha\left\{\mathbf{t}_{1}: \mathbf{L}_{1} ; \ldots ; \mathbf{t}_{n}: \mathbf{L}_{n}\right\} \prec_{2} s_{2}
\end{gathered}
$$

$(n \geq 0)$, where

- $\alpha$ is an aggregate name,

- each $\mathbf{t}_{i}$ is a tuple of terms,

- each $\mathbf{L}_{i}$ is a tuple of symbolic or arithmetic literals (if $\mathbf{L}_{i}$ is empty and $\mathbf{t}_{i}$ is nonempty then the preceding colon may be dropped),

- each of $\prec, \prec_{1}, \prec_{2}$ is one of the symbols (5),

- each of $s, s_{1}, s_{2}$ is a term.

For any aggregate atom $A$, the strings (8) are aggregate literals.

A literal is a conditional literal or an aggregate literal.

A choice expression is a string of the form $\{A\}$ where $A$ is an atom.

\subsection{Rules and Programs}

A rule is a string of the form

$$
H_{1} \vee \cdots \vee H_{k} \leftarrow B_{1} \wedge \cdots \wedge B_{m}
$$

\footnotetext{
7 This form of pooling is less general than what is allowed in the input language of GRINGO. For instance, $f(a ; b)$ is neither a term nor a pool.

${ }^{8}$ Semantically, the status of "double negations" in AG is the same as in logic programs with nested expressions (Lifschitz et al. 1999), where conjunction, disjunction, and negation can be nested arbitrarily. Dropping a double negation may change the meaning of a rule. For instance, the one-rule program $p \leftarrow$ not not $p$ has two stable models $\emptyset,\{p\}$ (see Section 4.1); the latter will disappear if we drop not not.

${ }^{9}$ In the input language of GRINGO, dropping the colon when $\mathbf{L}$ is empty is required.
} 
or of the form

$$
C \leftarrow B_{1} \wedge \cdots \wedge B_{m}
$$

$(k, m \geq 0)$, where each $H_{i}$ is a symbolic or arithmetic literal, ${ }^{10} C$ is a choice expression, and each $B_{j}$ is a literal. The expression $B_{1} \wedge \cdots \wedge B_{m}$ is the body of the rule; $H_{1} \vee \cdots \vee H_{k}$ is the head of (12); $C$ is the head of (13). If the body of a rule is empty and the head is not then the arrow can be dropped.

For instance, here are the first five rules of the program from Table 1 written in the syntax of AG:

$$
\begin{array}{ll}
R_{1}: & \{q(\overline{1} . . \bar{n}, \overline{1} . . \bar{n})\}, \\
R_{2}: & \leftarrow X=\overline{1} . . \bar{n} \wedge \text { not count }\{Y: q(X, Y)\}=\overline{1}, \\
R_{3}: & \leftarrow Y=\overline{1} . . \bar{n} \wedge \text { not count }\{X: q(X, Y)\}=\overline{1}, \\
R_{4}: & d 1(X, Y, X-Y+\bar{n}) \leftarrow X=\overline{1} . . \bar{n} \wedge Y=\overline{1} . . \bar{n}, \\
R_{5}: & d 2(X, Y, X+Y-\overline{1}) \leftarrow X=\overline{1} . . \bar{n} \wedge Y=\overline{1} . . \bar{n} .
\end{array}
$$

The other two rules use abbreviations introduced in the next section.

A program is a finite set of rules.

\section{Abbreviations}

Let $C$ be an expression of the form

$$
s_{1} \prec_{1} \alpha\left\{\mathbf{t}_{1}: L_{1}: \mathbf{L}_{1} ; \ldots ; \mathbf{t}_{n}: L_{n}: \mathbf{L}_{n}\right\} \prec_{2} s_{2}
$$

( $n \geq 0)$, where each $L_{i}$ is a symbolic literal of one of the forms

$$
p(\mathbf{t}) \quad \text { not } p(\mathbf{t}) \quad \text { not not } p(\mathbf{t})
$$

( $p$ is a symbolic or negated constant and $\mathbf{t}$ is a tuple of terms) and $\alpha, \mathbf{t}_{i}, \mathbf{L}_{i}, \prec_{1}, \prec_{2}, s_{1}$, and $s_{2}$ are as in the definition of an aggregate atom. Then a string of the form

$$
C \leftarrow B_{1} \wedge \cdots \wedge B_{m}
$$

( $m \geq 0$ ), where each $B_{j}$ is a literal, is shorthand for the set of rules consisting of the rule

$$
\leftarrow B_{1} \wedge \cdots \wedge B_{m} \wedge \text { not } s_{1} \prec_{1} \alpha\left\{\mathbf{t}_{1}: L_{1}, \mathbf{L}_{1} ; \ldots ; \mathbf{t}_{n}: L_{n}, \mathbf{L}_{n}\right\} \prec_{2} s_{2}
$$

and, for each $L_{i}$ in (14) such that $L_{i}$ is an atom, the rule

$$
\left\{L_{i}\right\} \leftarrow B_{1} \wedge \cdots \wedge B_{m} \wedge C_{i}
$$

where $C_{i}$ is the conjunction of the members of $\mathbf{L}_{i}$.

In both (17) and (18), the conjunction sign shown after $B_{m}$ should be dropped if $m=0$; in (18) it should also be dropped if $C_{i}$ is empty. The parts $s_{1} \prec_{1}$ and $\prec_{2} s_{2}$ in (14) are optional; if one of them is missing then it is dropped from (17) as well; if both are missing then rule (17) is dropped from the set altogether. If $m=0$ in (16) then the arrow can be dropped.

The term representations of literals (15) are the tuples

$$
\overline{0}, p(\mathbf{t}) \quad \overline{1}, p(\mathbf{t}) \quad \overline{2}, p(\mathbf{t})
$$

\footnotetext{
${ }^{10}$ In the input language of GRINGO, $H_{i}$ may be any conditional literal.
} 
of terms. (Each of them is indeed a tuple of terms, because $p(\mathbf{t})$ can be viewed as a term.)

Also viewed as an abbreviation is any expression of the form

$$
s_{1}\left\{L_{1}: \mathbf{L}_{1} ; \ldots ; L_{n}: \mathbf{L}_{n}\right\} s_{2}
$$

$(n>0)$, where $s_{1}, s_{2}$ are terms, each $L_{i}$ is a symbolic literal of one of the forms (15) that does not contain .. , and each $\mathbf{L}_{i}$ is a tuple of symbolic or arithmetic literals. ${ }^{11}$ Such an expression is understood differently depending on whether it occurs in the head or the body of a rule. In the head of a rule, (19) is understood as shorthand for an expression of the form (14):

$$
s_{1} \leq \operatorname{count}\left\{\mathbf{t}_{1}: L_{1}: \mathbf{L}_{1} ; \ldots ; \mathbf{t}_{n}: L_{n}: \mathbf{L}_{n}\right\} \leq s_{2}
$$

where $\mathbf{t}_{i}$ is the term representation of $L_{i}$. If either or both of the terms $s_{1}, s_{2}$ are missing, the abbreviation is understood in a similar way. (Note that choice expressions that do not contain .. are expressions of the form (19) where both $s_{1}$ and $s_{2}$ are missing, $n=1, L_{1}$ is of the form $p(\mathbf{t})$, and $\mathbf{L}_{1}$ is empty. In this case, we do not view (19) as an abbreviation.)

In the body of a rule (19) is understood as shorthand for the aggregate atom

$$
s_{1} \leq \operatorname{count}\left\{\mathbf{t}_{1}: L_{1}, \mathbf{L}_{1} ; \ldots ; \mathbf{t}_{n}: L_{n}, \mathbf{L}_{n}\right\} \leq s_{2}
$$

where $\mathbf{t}_{i}$ is the term representation of $L_{i} \cdot{ }^{12}$ If either of the terms $s_{1}, s_{2}$ in (19) is missing, the abbreviation is understood in a similar way.

These abbreviations can be used, for instance, to represent the last two rules of the program from Table 1 in the syntax of AG:

$$
\begin{aligned}
& \leftarrow D=\overline{1} . . \bar{n} * \overline{2}-\overline{1} \wedge \overline{2}\{q(X, Y): d 1(X, Y, D)\}, \\
& \leftarrow D=\overline{1} . . \bar{n} * \overline{2}-\overline{1} \wedge \overline{2}\{q(X, Y): d 2(X, Y, D)\} .
\end{aligned}
$$

Written out in full, these expressions become

$$
\begin{array}{ll}
R_{6}: & \leftarrow D=\overline{1} . . \bar{n} * \overline{2}-\overline{1} \wedge \overline{2} \leq \operatorname{count}\{\overline{0}, q(X, Y): q(X, Y), d 1(X, Y, D)\}, \\
R_{7}: & \leftarrow D=\overline{1} . . \bar{n} * \overline{2}-\overline{1} \wedge \overline{2} \leq \operatorname{count}\{\overline{0}, q(X, Y): q(X, Y), d 2(X, Y, D)\} .
\end{array}
$$

\section{Semantics of AG}

We will define the semantics of AG using a syntactic transformation $\tau$. The function $\tau$ converts rules into infinitary formulas formed from atoms of the form $p(\mathbf{t})$ or $\tilde{p}(\mathbf{t})$, where $p$ is a symbolic constant, and $\mathbf{t}$ is a tuple of precomputed terms. Then the stable models of a program will be defined in terms of stable model semantics of infinitary formulas in the sense of Truszczynski (2012), which is reviewed below.

\subsection{Review: Infinitary Propositional Formulas}

Let $\sigma$ be a propositional signature, that is, a set of propositional atoms. The sets $\mathcal{F}_{0}, \mathcal{F}_{1}, \ldots$ are defined as follows:

- $\mathcal{F}_{0}=\sigma$,

${ }^{11}$ To be precise, if $\mathbf{L}_{i}$ is empty then the colon after $L_{i}$ is dropped.

${ }^{12}$ If $\mathbf{L}_{i}$ is empty then the comma after $L_{i}$ in this expression should be dropped. 
- $\mathcal{F}_{i+1}$ is obtained from $\mathcal{F}_{i}$ by adding expressions $\mathcal{H}^{\wedge}$ and $\mathcal{H}^{\vee}$ for all subsets $\mathcal{H}$ of $\mathcal{F}_{i}$, and expressions $F \rightarrow G$ for all $F, G \in \mathcal{F}_{i}$.

The elements of $\bigcup_{i=0}^{\infty} \mathcal{F}_{i}$ are called (infinitary) formulas over $\sigma$.

In an infinitary formula, the symbols $\top$ and $\perp$ are understood as abbreviations for $\emptyset^{\wedge}$ and $\emptyset^{\vee}$ respectively; $\neg F$ stands for $F \rightarrow \perp$, and $F \leftrightarrow G$ stands for $(F \rightarrow G) \wedge(G \rightarrow F)$.

Subsets of a signature $\sigma$ will also be called its interpretations. The satisfaction relation between an interpretation and a formula is defined recursively as follows:

- For every atom $p$ from $\sigma, I \models p$ if $p \in I$.

- $I \models \mathcal{H}^{\wedge}$ if for every formula $F$ in $\mathcal{H}, I \models F$.

- $I \models \mathcal{H}^{\vee}$ if there is a formula $F$ in $\mathcal{H}$ such that $I \models F$.

- $I \models F \rightarrow G$ if $I \not \models F$ or $I \models G$.

We say that an interpretation satisfies a set $\mathcal{H}$ of formulas, or is a model of $\mathcal{H}$, if it satisfies every formula in $\mathcal{H}$. Two sets of formulas are equivalent if they have the same models.

The reduct $F^{I}$ of a formula $F$ w.r.t. an interpretation $I$ is defined as follows:

- For $p \in \sigma, p^{I}=\perp$ if $I \not \models p$; otherwise $p^{I}=p$.

- $\left(\mathcal{H}^{\wedge}\right)^{I}=\left\{G^{I} \mid G \in \mathcal{H}\right\}^{\wedge}$.

- $\left(\mathcal{H}^{\vee}\right)^{I}=\left\{G^{I} \mid G \in \mathcal{H}\right\}^{\vee}$.

- $(G \rightarrow H)^{I}=\perp$ if $I \not \models G \rightarrow H$; otherwise $(G \rightarrow H)^{I}=G^{I} \rightarrow H^{I}$.

An interpretation $I$ is a stable model of a set $\mathcal{H}$ of formulas if it is minimal w.r.t. set inclusion among the interpretations satisfying the reducts of all formulas from $\mathcal{H}$.

For instance, if $I=\emptyset$ then

$$
(\neg \neg p \rightarrow p)^{I}=(\neg \neg p)^{I} \rightarrow p^{I}=\perp \rightarrow \perp ;
$$

if $I=\{p\}$ then

$$
(\neg \neg p \rightarrow p)^{I}=(\neg \neg p)^{I} \rightarrow p^{I}=\neg(\neg p)^{I} \rightarrow p=\neg \perp \rightarrow p .
$$

In both cases, $I$ is a minimal model of the reduct. Consequently, both $\emptyset$ and $\{p\}$ are stable models of $\{\neg \neg p \rightarrow p\}$.

\subsection{Semantics of Terms and Pools}

A term is ground if it does not contain variables. The definition of "ground" for pools, symbolic literals, and arithmetic literals is the same. Semantically, every ground term $t$ represents a finite set of precomputed terms $[t]$, which is defined recursively:

- if $t$ is a numeral or a symbolic constant then $[t]$ is $\{t\}$;

- if $t$ is $f\left(t_{1}, \ldots, t_{n}\right)$ then $[t]$ is the set of terms $f\left(r_{1}, \ldots, r_{n}\right)$ for all $r_{1} \in\left[t_{1}\right], \ldots$, $r_{n} \in\left[t_{n}\right]$

- if $t$ is $\left(t_{1}+t_{2}\right)$ then $[t]$ is the set of numerals $\overline{n_{1}+n_{2}}$ for all integers $n_{1}, n_{2}$ such that $\overline{n_{1}} \in\left[t_{1}\right]$ and $\overline{n_{2}} \in\left[t_{2}\right]$; similarly when $t$ is $\left(t_{1}-t_{2}\right)$ or $\left(t_{1} \times t_{2}\right)$;

- if $t$ is $\left(t_{1} / t_{2}\right)$ then $[t]$ is the set of numerals $\overline{\left[n_{1} / n_{2}\right\rfloor}$ for all integers $n_{1}, n_{2}$ such that $\overline{n_{1}} \in\left[t_{1}\right], \overline{n_{2}} \in\left[t_{2}\right]$, and $n_{2} \neq 0$; 
- if $t$ is $\left(t_{1} . . t_{2}\right)$ then $[t]$ is the set of numerals $\bar{m}$ for all integers $m$ such that, for some integers $n_{1}, n_{2}$,

$$
\overline{n_{1}} \in\left[t_{1}\right], \quad \overline{n_{2}} \in\left[t_{2}\right], \quad n_{1} \leq m \leq n_{2} ;
$$

- if $t$ is $\left\langle t_{1}, \ldots, t_{n}\right\rangle$ then $[t]$ is the set of terms $\left\langle r_{1}, \ldots, r_{n}\right\rangle$ for all $r_{1} \in\left[t_{1}\right], \ldots$, $r_{n} \in\left[t_{n}\right]$.

This definition is extended to an arbitrary ground pool $P$; $[P]$ is a finite set of precomputed tuples:

- if $P$ is a tuple $t_{1}, \ldots, t_{n}$ of terms $(n \neq 1)$ then $[P]$ is the set of tuples $r_{1}, \ldots, r_{n}$ for all $r_{1} \in\left[t_{1}\right], \ldots, r_{n} \in\left[t_{n}\right]$

- if $P$ is a pool $\mathbf{t}_{1} ; \ldots ; \mathbf{t}_{n}(n>1)$ then $[P]$ is $\left[\mathbf{t}_{1}\right] \cup \cdots \cup\left[\mathbf{t}_{n}\right]$.

For instance, $[\overline{1} . . \bar{n}, \overline{1} . . \bar{n}]$ is the set $\{\bar{i}, \bar{j}: 1 \leq i, j \leq n\}$.

It is clear that if a ground term $t$ contains neither symbolic constants nor the symbols $<$ and $\rangle$ then every element of $[t]$ is a numeral. If a tuple $\mathbf{t}$ of ground terms is precomputed then $[\mathbf{t}]$ is $\{\mathbf{t}\}$. The set $[t]$ can be empty. For example, $[1 . .0]=[1 / 0]=[1+a]=\emptyset$.

About a tuple of terms that does not contain .. we say that it is interval-free. It is clear that if a tuple $\mathbf{t}$ of ground terms is interval-free then the cardinality of the set $[\mathbf{t}]$ is at most 1 .

\subsection{Semantics of Arithmetic and Symbolic Literals}

For any ground (symbolic or arithmetic) literal $L$ we will define two translations, $\tau_{\wedge} L$ and $\tau_{\vee} L$. The specific translation function applied to an occurrence of a symbolic or arithmetic literal in a rule depends on the context, as we will see in the following sections.

We will first consider symbolic literals. For any ground atom $A$,

- if $A$ is $p(P)$ then $\tau_{\wedge} A$ is the conjunction of atoms $p(\mathbf{t})$ over all tuples $\mathbf{t}$ in $[P]$, and $\tau_{\vee} A$ is the disjunction of these atoms;

- if $A$ is $\tilde{p}(P)$ then $\tau_{\wedge} A$ is the conjunction of atoms $\tilde{p}(\mathbf{t})$ over all tuples $\mathbf{t}$ in $[P]$, and $\tau_{\vee} A$ is the disjunction of these atoms;

- $\tau_{\wedge}($ not $A)$ is $\neg \tau_{\vee} A$, and $\tau_{\vee}($ not $A)$ is $\neg \tau_{\wedge} A$;

- $\tau_{\wedge}($ not not $A)$ is $\neg \neg \tau_{\wedge} A$, and $\tau_{\vee}($ not not $A)$ is $\neg \neg \tau_{\vee} A$.

The definitions of $\tau_{\wedge}$ and $\tau_{\vee}$ for arithmetic literals are as follows:

- $\tau_{\wedge}\left(t_{1} \prec t_{2}\right)$ is $\top$ if the relation $\prec$ holds between the terms $r_{1}$ and $r_{2}$ for all $r_{1} \in\left[t_{1}\right]$ and $r_{2} \in\left[t_{2}\right]$, and $\perp$ otherwise;

- $\tau_{\vee}\left(t_{1} \prec t_{2}\right)$ is $\top$ if the relation $\prec$ holds between the terms $r_{1}$ and $r_{2}$ for some $r_{1}, r_{2}$ such that $r_{1} \in\left[t_{1}\right]$ and $r_{2} \in\left[t_{2}\right]$, and $\perp$ otherwise.

For instance, $\tau_{\vee} p(\overline{2} . . \overline{4})$ is $p(\overline{2}) \vee p(\overline{3}) \vee p(\overline{4})$, and $\tau_{\vee}(\overline{2}=\overline{2} . . \overline{4})$ is $\top$.

For any tuple $\mathbf{L}$ of ground literals, $\tau_{\vee} \mathbf{L}$ stands for the conjunction of the formulas $\tau_{\vee} L$ for all members $L$ of $\mathbf{L}$. The expressions $\tau_{\wedge} \perp$ and $\tau_{\vee} \perp$ both stand for $\perp$.

It is clear that if $A$ has the form $p(\mathbf{t})$ or $\tilde{p}(\mathbf{t})$, where $\mathbf{t}$ is a tuple of precomputed terms, then each of the formulas $\tau_{\wedge} A$ and $\tau_{\vee} A$ is $A$. 


\subsection{Semantics of Choice Expressions}

The result of applying $\tau$ to a choice expression $\{p(P)\}$ is the conjunction of the formulas $p(\mathbf{t}) \vee \neg p(\mathbf{t})$ over all tuples $\mathbf{t}$ in $[P]$. Similarly, the result of applying $\tau$ to a choice expression $\{\tilde{p}(P)\}$ is the conjunction of the formulas $\tilde{p}(\mathbf{t}) \vee \neg \tilde{p}(\mathbf{t})$ over all tuples $\mathbf{t}$ in $[P]$.

For instance, the result of applying $\tau$ to rule $R_{1}$ (see Section 2.3) is

$$
\bigwedge_{1 \leq i, j \leq n}(q(\bar{i}, \bar{j}) \vee \neg q(\bar{i}, \bar{j})) .
$$

\subsection{Global Variables}

About a variable we say that it is global

- in a conditional literal $H: \mathbf{L}$, if it occurs in $H$ but does not occur in $\mathbf{L}$;

- in an aggregate literal $A$, not $A$, or not not $A$, where $A$ is of one of the forms (9)-(11), if it occurs in $s, s_{1}$, or $s_{2}$;

- in a rule (12), if it is global in at least one of the expressions $H_{i}, B_{j}$;

- in a rule (13), if it occurs in $C$ or is global in at least one of the expressions $B_{j}$.

An instance of a rule $R$ is any rule that can be obtained from $R$ by substituting precomputed terms for all global variables. ${ }^{13}$ A literal or a rule is closed if it has no global variables. It is clear that any instance of a rule is closed.

For example, $X$ is global in the rule $R_{2}$ from Section 2.3, so that the instances of $R_{2}$ are rules of the form

$$
\leftarrow r=\overline{1} . . \bar{n} \wedge \text { not count }\{Y: q(r, Y)\}=\overline{1}
$$

for all precomputed terms $r$. The variables $X$ and $Y$ are global in $R_{4}$; instances of $R_{4}$ are

$$
d l(r, s, r-s+\bar{n}) \leftarrow r=\overline{1} . . \bar{n} \wedge s=\overline{1} . . \bar{n}
$$

for all precomputed terms $r$ and $s$.

\subsection{Semantics of Conditional Literals}

If $t$ is a term, $\mathbf{x}$ is a tuple of distinct variables, and $\mathbf{r}$ is a tuple of terms of the same length as $\mathbf{x}$, then the term obtained from $t$ by substituting $\mathbf{r}$ for $\mathbf{x}$ will be denoted by $t_{\mathbf{r}}^{\mathbf{x}}$. Similar notation will be used for the result of substituting $\mathbf{r}$ for $\mathbf{x}$ in expressions of other kinds, such as literals and tuples of literals.

The result of applying $\tau$ to a closed conditional literal $H: \mathbf{L}$ is the conjunction of the formulas

$$
\tau_{\vee}\left(\mathbf{L}_{\mathbf{r}}^{\mathbf{x}}\right) \rightarrow \tau_{\vee}\left(H_{\mathbf{r}}^{\mathbf{x}}\right)
$$

where $\mathbf{x}$ is the list of variables occurring in $H: \mathbf{L}$, over all tuples $\mathbf{r}$ of precomputed terms of the same length as $\mathbf{x}$.

13 This definition differs slightly from that given by Harrison et al. (2014, Section 3.3). There, substitutions that yield symbolic constants in the scope of arithmetical operators do not form instances. In a similar way, we treat variables in conditional literals and aggregate literals (Sections 4.6 and 4.7) differently than how they are treated by Harrison et al. (2014). 
For instance, the result of applying $\tau$ to the arithmetic literal $r=\overline{1} . . \bar{n}$, where $r$ is a precomputed term, is $\tau_{\vee}(\epsilon) \rightarrow \tau_{\vee}(r=\overline{1} . . \bar{n})$, where $\epsilon$ is the tuple of length 0 . The antecedent of this implication is $\top$. The consequent is $\top$ if $r$ is one of the numerals $\overline{1}, \ldots, \bar{n}$ and $\perp$ otherwise.

\subsection{Semantics of Aggregate Literals}

In this section, the semantics of ground aggregates proposed by Ferraris (2005, Section 4.1) is adapted to closed aggregate literals. Let $E$ be a closed aggregate atom of one of the forms (9)-(11), and let $\mathbf{x}_{i}$ be the list of variables occurring in $\mathbf{t}_{i}: \mathbf{L}_{i}(1 \leq i \leq n)$. By $A_{i}$ we denote the set of tuples $\mathbf{r}$ of precomputed terms of the same length as $\mathbf{x}_{i}$. By $A$ we denote the set $\left\{(i, \mathbf{r}): i \in\{1, \ldots, n\}, \mathbf{r} \in A_{i}\right\}$.

Let $\Delta$ be a subset of $A$. Then by $[\Delta]$ we denote the union of the sets $\left[\left(\mathbf{t}_{i}\right)_{\mathbf{r}}^{\mathbf{x}_{i}}\right]$ for all pairs $(i, \mathbf{r}) \in \Delta$. We say that $\Delta$ justifies $E$ if

- $E$ is of the form (9) and the relation $\prec$ holds between $\widehat{\alpha}[\Delta]$ and $t$ for at least one precomputed term $t \in[s]$, or

- $E$ is of the form (10) and the relation $\prec$ holds between $t$ and $\widehat{\alpha}[\Delta]$ for at least one precomputed term $t \in[s]$, or

- $E$ is of the form (11), the relation $\prec_{1}$ holds between $t_{1}$ and $\widehat{\alpha}[\Delta]$ for at least one precomputed term $t_{1} \in\left[s_{1}\right]$, and the relation $\prec_{2}$ holds between $\widehat{\alpha}[\Delta]$ and $t_{2}$ for at least one precomputed term $t_{2} \in\left[s_{2}\right]$.

We define $\tau E$ as the conjunction of the implications

$$
\bigwedge_{(i, \mathbf{r}) \in \Delta} \tau_{\vee}\left(\left(\mathbf{L}_{i}\right)_{\mathbf{r}}^{\mathbf{x}_{i}}\right) \rightarrow \bigvee_{(i, \mathbf{r}) \in A \backslash \Delta} \tau_{\vee}\left(\left(\mathbf{L}_{i}\right)_{\mathbf{r}}^{\mathbf{x}_{i}}\right)
$$

over all sets $\Delta$ that do not justify $E$.

For instance, if $E$ is count $\{p(X): p(X)\}>0$ then $\tau E$ is the implication expressing that $p(r)$ holds for at least one precomputed term $r$ :

$$
\top \rightarrow \bigvee_{r} p(r)
$$

If $L$ is an aggregate literal of the form not $E$ where $E$ is an aggregate atom then $\tau L$ is $\neg \tau E$; if $L$ is of the form not not $E$ then $\tau L$ is $\neg \neg \tau E$.

\subsection{Semantics of Rules and Programs}

For any rule $R$ of form (12), $\tau R$ stands for the set of the formulas

$$
\tau B_{1} \wedge \cdots \wedge \tau B_{m} \rightarrow \tau_{\wedge} H_{1} \vee \cdots \vee \tau_{\wedge} H_{k}
$$

for all instances (12) of $R$. For a rule of form (13), $\tau R$ stands for the set of the formulas

$$
\tau B_{1} \wedge \cdots \wedge \tau B_{m} \rightarrow \tau C
$$

for all instances (13) of $R$. For any program $\Pi, \tau \Pi$ stands for the union of the sets $\tau R$ for all rules $R$ of $\Pi$.

A stable model of a program $\Pi$ is any stable model of $\tau \Pi$ (in the sense of Section 4.1) that does not contain any pair of atoms of the form $p(\mathbf{t}), \tilde{p}(\mathbf{t})$. 


\section{Simplifying $\tau \Pi$}

When we investigate the stable models of an AG program, it is often useful to simplify the formulas obtained by applying transformation $\tau$ to its rules. By simplifying an infinitary propositional formula we mean turning it into a strongly equivalent formula that has simpler syntactic structure. The definition of strong equivalence, introduced by Lifschitz et al. (2001), is extended to infinitary formulas by Harrison et al. (2015). Corollary 1 from that paper shows that the stable models of an infinitary formula are not affected by simplifying its parts.

Proofs of the theorems stated in this section are outlined in the electronic appendix.

\subsection{Monotone and Anti-Monotone Aggregate Atoms}

When a rule contains aggregate atoms, we can sometimes simplify the implications (22) in the corresponding infinitary formula using the theorems on monotone and anti-monotone aggregates from Harrison et al. (2014, Section 6.1). The monotonicity or non-monotonicity of an aggregate atom (9) can sometimes be established simply by looking at its aggregate name $\alpha$ and its relation symbol $\prec$. If $\alpha$ is one of the symbols count, sum + , max, then (9) is monotone when $\prec$ is $<$ or $\leq$, and anti-monotone when $\prec$ is $>$ or $\geq$. It is the other way around if $\alpha$ is $\min$.

Our semantics of aggregates is somewhat different from that adopted by Harrison et al. (2014, Section 3.5), as explained in Footnote 6 (and also in view of the difference in the treatment of variables discussed in Footnote 13). Nevertheless, the statements and proofs of the two theorems mentioned above remain essentially the same in the framework of AG. The theorems show that the antecedent in (22) can be dropped if $E$ is monotone, and that the consequent can be replaced by $\perp$ if $E$ is anti-monotone. These simplifications produce strongly equivalent formulas.

\subsection{Eliminating Equality from Aggregate Atoms}

If $\prec$ in an aggregate atom (9) is = then the following proposition can be useful, in combination with the facts reviewed in Section 5.1:

Theorem 1

Let $E$ be a closed aggregate atom of the form

$$
\alpha\left\{\mathbf{t}_{1}: \mathbf{L}_{1} ; \ldots ; \mathbf{t}_{n}: \mathbf{L}_{n}\right\}=s,
$$

where $s$ is an interval-free term. If $E_{\leq}$is

$$
\alpha\left\{\mathbf{t}_{1}: \mathbf{L}_{1} ; \ldots ; \mathbf{t}_{n}: \mathbf{L}_{n}\right\} \leq s
$$

and $E_{\geq}$is

$$
\alpha\left\{\mathbf{t}_{1}: \mathbf{L}_{1} ; \ldots ; \mathbf{t}_{n}: \mathbf{L}_{n}\right\} \geq s,
$$

then $\tau E$ is strongly equivalent to $\tau E_{\leq} \wedge \tau E_{\geq}$.

Without the assumption that $s$ is interval-free the assertion of Theorem 1 would be incorrect: if $E$ is count $\{a: p\}=(\overline{0} . . \overline{1}) \times \overline{2}$ then $\tau E$ is $p \rightarrow \perp$, but each of the formulas $\tau E_{\leq}, \tau E_{\geq}$is the empty conjunction $\top$. 


\subsection{Properties of Counting}

For any set $S$, by $|S|$ we denote the cardinality of $S$ if $S$ is finite, and $\infty$ otherwise.

Theorem 2

For any closed aggregate atom $E$ of the form

$$
\operatorname{count}\left\{\mathbf{t}_{1}: \mathbf{L}_{1} ; \ldots ; \mathbf{t}_{n}: \mathbf{L}_{n}\right\} \geq \bar{m}
$$

where $m$ is an integer and each $\mathbf{t}_{i}$ is interval-free, $\tau E$ is strongly equivalent to

$$
\bigvee_{\substack{\Delta \subseteq A \\|[\Delta]|=m}} \bigwedge_{(i, \mathbf{r}) \in \Delta} \tau_{\vee}\left(\left(\mathbf{L}_{i}\right)_{\mathbf{r}}^{\mathbf{x}_{i}}\right) .
$$

Theorem 3

For any closed aggregate atom $E$ of the form

$$
\operatorname{count}\left\{\mathbf{t}_{1}: \mathbf{L}_{1} ; \ldots ; \mathbf{t}_{n}: \mathbf{L}_{n}\right\} \leq \bar{m}
$$

where $m$ is an integer and each $\mathbf{t}_{i}$ is interval-free, $\tau E$ is strongly equivalent to

$$
\bigwedge_{\substack{\Delta \subseteq A \\|[\Delta]|=m+1}} \neg \bigwedge_{(i, \mathbf{r}) \in \Delta} \tau_{\vee}\left(\left(\mathbf{L}_{i}\right)_{\mathbf{r}}^{\mathbf{x}_{i}}\right) .
$$

Without the assumption that each $\mathbf{t}_{i}$ is interval-free the assertions of the theorems would be incorrect. For instance, if $E$ is $\operatorname{count}\{\overline{1} . . \overline{2}: p\} \geq \overline{1}$ then $\tau E$ is $\top \rightarrow p$, and (23) is $\perp$.

In the special (but common) case when $E$ has the form $\operatorname{count}\{\mathbf{x}: \mathbf{L}\} \geq \bar{m}$, where $\mathbf{x}$ is a tuple of variables and each variable occurring in $\mathbf{L}$ occurs also in $\mathbf{x}$, the condition $|[\Delta]|=m$ in (23) can be replaced by $|\Delta|=m$. Indeed, in this case $\Delta$ and $[\Delta]$ have the same cardinality because $[\Delta]$ is the set of tuples $\mathbf{r}$ of terms such that $(1, \mathbf{r}) \in \Delta$. Similarly, the condition $|[\Delta]|=m+1$ in (24) can be replaced by $|\Delta|=m+1$ if $E$ has the form $\operatorname{count}\{\mathbf{x}: \mathbf{L}\} \leq \bar{m}$.

\section{Conclusion}

We proposed a definition of stable models for programs in the language AG and stated a few theorems that facilitate reasoning about them. This definition can be viewed as a specification for the answer set system CLINGO (see Footnote 1) and other systems with the same input language. If such a system terminates given the ASCII representation of an AG program $\Pi$ as input, and produces neither error messages nor warnings, then its output is expected to represent the stable models of $\Pi$.

\section{Acknowledgements}

We are grateful to the anonymous referees for useful comments. 


\section{Abstract Gringo}

\section{References}

Calimeri, F., Faber, W., Gebser, M., Ianni, G., Kaminski, R., Krennwallner, T., LeOne, N., RiCCA, F., AND Schaub, T. 2012. ASP-Core-2: Input language format. Available at https://www.mat.unical.it/aspcomp2013/files/ASP-CORE-2.0.pdf.

FERraris, P. 2005. Answer sets for propositional theories. In Proceedings of International Conference on Logic Programming and Nonmonotonic Reasoning (LPNMR). 119-131.

FERRARIS, P. AND Lifschitz, V. 2005. Weight constraints as nested expressions. Theory and Practice of Logic Programming 5, 1-2, 45-74.

Gebser, M., Kaminski, R., Kaufmann, B., And Schaub, T. 2011. Challenges in answer set solving. In Logic programming, knowledge representation, and nonmonotonic reasoning. Springer, 74-90.

Harrison, A., Lifschitz, V., Pearce, D., And Valverde, A. 2015. Infinitary equilibrium logic and strong equivalence. In Proceedings of International Conference on Logic Programming and Nonmonotonic Reasoning (LPNMR). http://www.cs.utexas.edu/users/vl/ papers/iel_lpnmr.pdf; to appear.

HARrison, A., Lifschitz, V., AND YANG, F. 2014. The semantics of Gringo and infinitary propositional formulas. In Proceedings of International Conference on Principles of Knowledge Representation and Reasoning $(K R)$.

Lifschitz, V., Pearce, D., And Valverde, A. 2001. Strongly equivalent logic programs. ACM Transactions on Computational Logic 2, 526-541.

Lifschitz, V., TANG, L. R., AND TURner, H. 1999. Nested expressions in logic programs. Annals of Mathematics and Artificial Intelligence 25, 369-389.

TruszCZYNSKI, M. 2012. Connecting first-order ASP and the logic FO(ID) through reducts. In Correct Reasoning: Essays on Logic-Based AI in Honor of Vladimir Lifschitz, E. Erdem, J. Lee, Y. Lierler, and D. Pearce, Eds. Springer, 543-559. 


\title{
Online appendix for the paper \\ Abstract Gringo
}

\section{published in Theory and Practice of Logic Programming}

\author{
MARTIN GEBSER \\ Aalto University, HIIT, Finland \\ University of Potsdam, Germany \\ gebser@cs.uni-potsdam.de \\ AMELIA HARRISON \\ Univeristy of Texas at Austin, USA \\ ameliaj@cs.utexas.edu \\ ROLAND KAMINSKI \\ University of Potsdam, Germany \\ kaminski@cs.uni-potsdam.de \\ VLADIMIR LIFSCHITZ \\ Univeristy of Texas at Austin, USA \\ vl@cs.utexas.edu

\section{TORSTEN SCHAUB} \\ University of Potsdam, Germany \\ INRIA Rennes, France \\ torsten@cs.uni-potsdam.de
}

\section{Proofs of Theorems 1-3}

\section{Proof of Theorem 1}

Because $s$ is interval-free, $[s]$ is either empty or a singleton. Case 1: The set $[s]$ is empty. Then no set $\Delta \subseteq A$ justifies any of the aggregate atoms $E, E_{\leq}, E_{\geq}$. Consequently, each of the formulas $\tau E, \tau E_{\leq}, \tau E_{\geq}$is the conjunction of implications (22) for all sets $\Delta \subseteq A$. Case 2: The set $[s]$ is a singleton set $\{t\}$, where $t$ is a precomputed term. Then, we will show that the set of conjunctive terms of $\tau E$ is the union of the sets of conjunctive terms of $\tau E_{\leq}$and $\tau E_{\geq}$. For any subset $\Delta$ of $A$, 
(22) is a conjunctive term of $\tau E$

iff $\Delta$ does not justify $E$

iff $\widehat{\alpha}[\Delta] \neq t$

iff $\widehat{\alpha}[\Delta]<t$ or $\widehat{\alpha}[\Delta]>t$

iff $\Delta$ does not justify $E_{\geq}$or $\Delta$ does not justify $E_{\leq}$

iff (22) is a conjunctive term of $\tau E_{\geq}$or of $\tau E_{\leq}$

iff (22) is a conjunctive term of $\tau E_{\leq} \wedge \tau E_{\geq}$.

\section{Proof of Theorem 2}

Since $E$ is monotone, the antecedent of (22) can be dropped (Section 5.1), so that $\tau E$ is strongly equivalent to

$$
\bigwedge_{\substack{\Delta \subseteq A \\|[\Delta]|<m}} \bigvee_{(i, \mathbf{r}) \in A \backslash \Delta} \tau_{\vee}\left(\left(\mathbf{L}_{i}\right)_{\mathbf{r}}^{\mathbf{x}_{i}}\right)
$$

To derive (25) from (22) in $\mathrm{HT}^{\infty}$, assume (23). We will reason by cases, with one case corresponding to each disjunctive term

$$
\bigwedge_{(i, \mathbf{r}) \in \Delta} \tau_{\vee}\left(\left(\mathbf{L}_{i}\right)_{\mathbf{r}}^{\mathbf{x}_{i}}\right)
$$

of (23). Let $\Delta^{\prime}$ be a subset of $A$ such that $\left|\left[\Delta^{\prime}\right]\right|<m$. We will show that the conjunctive term of (25) corresponding to $\Delta^{\prime}$ can be derived from (26). Since

$$
\left|\left[\Delta^{\prime}\right]\right|<m=|[\Delta]|,
$$

there exists a pair $(i, \mathbf{r})$ that is an element of $\Delta$ but not an element of $\Delta^{\prime}$. Indeed, if $\Delta \subseteq \Delta^{\prime}$ then $[\Delta] \subseteq\left[\Delta^{\prime}\right]$, which contradicts (27). Since $(i, \mathbf{r}) \in \Delta$, from (26) we can derive $\tau_{\vee}\left(\left(\mathbf{L}_{i}\right)_{\mathbf{r}}^{\mathbf{x}_{i}}\right)$. Since $(i, \mathbf{r}) \in A \backslash \Delta^{\prime}$, we can further derive

$$
\bigvee_{(i, \mathbf{r}) \in A \backslash \Delta^{\prime}} \tau_{\vee}\left(\left(\mathbf{L}_{i}\right)_{\mathbf{r}}^{\mathbf{x}_{i}}\right)
$$

It follows that each conjunctive term of (25) can be derived from (26).

We will prove by induction on $m$ that (23) can be derived from (25) in $\mathrm{HT}^{\infty}$. Base case: when $m=0$ the disjunctive term of (23) corresponding to the empty $\Delta$ is $T$. Inductive step: assume that (23) can be derived from (25), and assume

$$
\bigwedge_{\substack{\Delta \subseteq A \\|[\Delta]|<m+1}} \bigvee_{(i, \mathbf{r}) \in A \backslash \Delta} \tau_{\vee}\left(\left(\mathbf{L}_{i}\right)_{\mathbf{r}}^{\mathbf{x}_{i}}\right) .
$$

From (28) we can derive (25), and consequently (23). Now we reason by cases, with one case corresponding to each disjunctive term of (23). Assume

$$
\bigwedge_{(i, \mathbf{r}) \in \Sigma} \tau_{\vee}\left(\left(\mathbf{L}_{i}\right)_{\mathbf{r}}^{\mathbf{x}_{i}}\right)
$$

where $\Sigma$ is a subset of $A$ such that $|[\Sigma]|=m$. Consider the set

$$
\Sigma^{\prime}=\left\{(i, \mathbf{r}):\left[\left(\mathbf{t}_{i}\right)_{\mathbf{r}}^{\mathbf{x}_{i}}\right] \subseteq[\Sigma]\right\}
$$

By the definition of $[\Sigma]$, for any $(i, \mathbf{r}) \in \Sigma,\left[\left(\mathbf{t}_{i}\right)_{\mathbf{r}}^{\mathbf{x}_{i}}\right] \subseteq[\Sigma]$. So $\Sigma \subseteq \Sigma^{\prime}$. It follows that 
$[\Sigma] \subseteq\left[\Sigma^{\prime}\right]$. On the other hand,

$$
\left[\Sigma^{\prime}\right]=\bigcup_{(i, \mathbf{r}) \in \Sigma^{\prime}}\left[\left(\mathbf{t}_{i}\right)_{\mathbf{r}}^{\mathbf{x}_{i}}\right]=\bigcup_{(i, \mathbf{r}):\left[\left(\mathbf{t}_{i}\right)_{\mathbf{r}}^{\mathbf{x}_{i}} \subseteq \subseteq[\Sigma]\right.}\left[\left(\mathbf{t}_{i}\right)_{\mathbf{r}}^{\mathbf{x}_{i}}\right] \subseteq[\Sigma] .
$$

Consequently $[\Sigma]=\left[\Sigma^{\prime}\right]$, and $\left|\left[\Sigma^{\prime}\right]\right|=|[\Sigma]|=m$. From $(28)$,

$$
\bigvee_{(i, \mathbf{r}) \in A \backslash \Sigma^{\prime}} \tau_{\vee}\left(\left(\mathbf{L}_{i}\right)_{\mathbf{r}}^{\mathbf{x}_{i}}\right)
$$

Again, we reason by cases, with one case corresponding to each disjunctive term of (30). Assume $\tau_{\vee}\left(\left(\mathbf{L}_{j}\right)_{\mathbf{s}}^{\mathbf{x}_{j}}\right)$, where $(j, \mathbf{s}) \in A \backslash \Sigma^{\prime}$. Combining assumption (29) and $\tau_{\vee}\left(\left(\mathbf{L}_{j}\right)_{\mathbf{s}}^{\mathbf{x}_{j}}\right)$, we derive

$$
\bigwedge_{(i, \mathbf{r}) \in \Sigma \cup\{(j, \mathbf{s})\}} \tau_{\vee}\left(\left(\mathbf{L}_{i}\right)_{\mathbf{r}}^{\mathbf{x}_{i}}\right) .
$$

Consider the set $[\Sigma \cup\{(j, \mathbf{s})\}]$, that is,

$$
[\Sigma] \cup\left[\left(\mathbf{t}_{j}\right)_{\mathbf{s}}^{\mathbf{x}_{j}}\right]
$$

Recall that the cardinality of $[\Sigma]$ is $m$. Since $\mathbf{t}_{j}$ is interval-free, the cardinality of $\left[\left(\mathbf{t}_{j}\right)_{\mathbf{s}}^{\mathbf{x}_{j}}\right]$ is at most 1 . Furthermore, since $(j, \mathbf{s}) \notin \Sigma^{\prime}$ it follows that

$$
\left[\left(\mathbf{t}_{j}\right)_{\mathbf{s}}^{\mathbf{x}_{j}}\right] \nsubseteq[\Sigma]
$$

so that $\left[\left(\mathbf{t}_{j}\right)_{\mathbf{s}}^{\mathbf{x}_{j}}\right]$ is nonempty. Consequently, the set is a singleton, and therefore $[\Sigma]$ is disjoint from it. It follows that the cardinality of (32) is $m+1$. So from (31) we can derive

$$
\bigvee_{\substack{\Delta \subseteq A \\|[\Delta]|=m+1}} \bigwedge_{(i, \mathbf{r}) \in \Delta} \tau_{\vee}\left(\left(\mathbf{L}_{i}\right)_{\mathbf{r}}^{\mathbf{x}_{i}}\right)
$$

\section{Proof of Theorem 3}

Since the consequent of (22) can be replaced in this case by $\perp, \tau E$ is strongly equivalent to

$$
\bigwedge_{\substack{\Delta \subseteq A \\|[\Delta]|>m}} \neg \bigwedge_{(i, \mathbf{r}) \in \Delta} \tau_{\vee}\left(\left(\mathbf{L}_{i}\right)_{\mathbf{r}}^{\mathbf{x}_{i}}\right)
$$

Every conjunctive term of (24) is a conjunctive term of (33). To derive (33) from (24), consider a set $\Delta$ such that $|[\Delta]|>m$. Let $f(i, \mathbf{r})$ stand for the set $\left[\left(\mathbf{t}_{i}\right)_{\mathbf{r}}^{\mathbf{x}_{i}}\right]$. Since each $\mathbf{t}_{i}$ is interval-free, this set is either empty or a singleton. Let $\mathbf{s}_{1}, \ldots, \mathbf{s}_{m+1}$ be $m+1$ distinct elements of $[\Delta]$. Choose elements $\left(i_{1}, \mathbf{r}_{1}\right), \ldots,\left(i_{m+1}, \mathbf{r}_{m+1}\right)$ of $\Delta$ such that each $s_{k}$ belongs to $f\left(i_{k}, \mathbf{r}_{k}\right)$, and let $\Delta^{\prime}$ be $\left\{\left(i_{1}, \mathbf{r}_{1}\right), \ldots,\left(i_{m+1}, \mathbf{r}_{m+1}\right)\right\}$. The cardinality of $\left[\Delta^{\prime}\right]$ is at least $m+1$, because this set includes $\mathbf{s}_{1}, \ldots, \mathbf{s}_{m+1}$. On the other hand, it is at most $m+1$, because this set is the union of $m+1$ sets of cardinality at most 1 . Consequently, $\left|\left[\Delta^{\prime}\right]\right|=m+1$. From $(24)$ we can conclude in $\mathrm{HT}^{\infty}$ that

$$
\neg \bigwedge_{(i, \mathbf{r}) \in \Delta^{\prime}} \tau_{\vee}\left(\left(\mathbf{L}_{i}\right)_{\mathbf{r}}^{\mathbf{x}_{i}}\right) .
$$

Then the conjunctive term

$$
\neg \bigwedge_{(i, \mathbf{r}) \in \Delta} \tau_{\vee}\left(\left(\mathbf{L}_{i}\right)_{\mathbf{r}}^{\mathbf{x}_{i}}\right)
$$


of (33) follows, because $\Delta^{\prime} \subseteq \Delta$.

\section{Correctness of the $n$-Queens Program}

In this section, we prove the correctness of the program $K$, consisting of rules $R_{1}, \ldots, R_{7}$ (Sections 2.3 and 3).

The $n$-queens problem involves placing $n$ queens on an $n \times n$ chess board such that no two queens threaten each other. We will represent squares by pairs of integers $(i, j)$ where $1 \leq i, j \leq n$. Two squares $\left(i_{1}, j_{1}\right)$ and $\left(i_{2}, j_{2}\right)$ are said to be in the same row if $i_{1}=i_{2}$; in the same column if $j_{1}=j_{2}$; and in the same diagonal if $\left|i_{1}-i_{2}\right|=\left|j_{1}-j_{2}\right|$. A set $Q$ of $n$ squares is a solution to the $n$-queens problem if no two elements of $Q$ are in the same row, in the same column, or in the same diagonal.

For any stable model $I$ of $K$, by $Q_{I}$ we denote the set of pairs $(i, j)$ such that $q(\bar{i}, \bar{j}) \in I$.

Theorem 4

For each stable model $I$ of $K, Q_{I}$ is a solution to the $n$-queens problem. Furthermore, for each solution $Q$ to the $n$-queens problem there is exactly one stable model $I$ of $K$ such that $Q_{I}=Q$.

\section{Review: Supported Models and Constraints}

We start by reviewing two familiar facts that will be useful in proving Theorem 4 .

An infinitary program is a conjunction of (possibly infinitely many) infinitary formulas of the form $G \rightarrow A$, where $A$ is an atom. We say that an interpretation $I$ is supported by an infinitary program $\Pi$ if each atom $A$ from $I$ is the consequent of a conjunctive term $G \rightarrow A$ of $\Pi$ such that $I \models G$. Lifschitz and Yang (2013) give a condition, "tightness on an interpretation," under which the stable models of an infinitary program are identical to its supported models. Proposition 1 below gives a simpler condition of this kind that is sufficient for our purposes.

We say that an atom $A$ occurs nonnegated in a formula $F$ if

- $F$ is $A$, or

- $F$ is of the form $\mathcal{H}^{\wedge}$ or $\mathcal{H}^{\vee}$ and $A$ occurs nonnegated in at least one element of $\mathcal{H}$, or

- $F$ is of the form $G \rightarrow H$, where $H$ is different from $\perp$, and $A$ occurs nonnegated in $G$ or in $H$.

It is clear, for instance, that no atom occurs nonnegated in a formula of the form $\neg F$.

The positive dependency graph of an infinitary program $\Pi$ is the directed graph containing a vertex for each atom occuring in $\Pi$, and an edge from $A$ to $B$ for every conjunctive term $G \rightarrow A$ of $\Pi$ and every atom $B$ that occurs nonnegated in $G$. We say that an infinitary program $\Pi$ is extratight if the positive dependency graph of $\Pi$ contains no infinite paths.

The following fact is immediate from (Lifschitz and Yang 2013, Lemma 2).

Proposition 1

For any model $I$ of an extratight infinitary program $\Pi, I$ is stable iff $I$ is supported by $\Pi$. 
A constraint is an infinitary formula of the form $\neg F$ (which is shorthand for $F \rightarrow \perp$ ). The following theorem is a straightforward generalization of Proposition 4 from (Ferraris and Lifschitz 2005).

Proposition 2

Let $\mathcal{H}_{1}$ be a set of infinitary formulas and $\mathcal{H}_{2}$ be a set of constraints. A set $I$ of atoms is a stable model of $\mathcal{H}_{1} \cup \mathcal{H}_{2}$ iff $I$ is a stable model of $\mathcal{H}_{1}$ and satisfies all formulas in $\mathcal{H}_{2}$.

Proof

Case 1: Every formula in $\mathcal{H}_{1} \cup \mathcal{H}_{2}$ is satisfied by $I$. For each formula $\neg F$ in $\mathcal{H}_{2}, I$ does not satisfy $F$. So the reduct of each formula in $\mathcal{H}_{2}$ w.r.t. $I$ is $\neg \perp$. It follows that the set of reducts of all formulas in $\mathcal{H}_{1} \cup \mathcal{H}_{2}$ is satisfied by the same interpretations as the set of reducts of all formulas in $\mathcal{H}_{1}$. Consequently, $I$ is minimal among the sets satisfying the reducts of all formulas from $\mathcal{H}_{1} \cup \mathcal{H}_{2}$ iff it is minimal among the sets satisfying the reducts of all formulas from $\mathcal{H}_{1}$. Case 2: Some formula $F$ in $\mathcal{H}_{1} \cup \mathcal{H}_{2}$ is not satisfied by $I$. Then $I$ is not a stable model of $\mathcal{H}_{1} \cup \mathcal{H}_{2}$. If $F \in \mathcal{H}_{1}$ then $I$ is not a stable model of $\mathcal{H}_{1}$. Otherwise, it is not true that $I$ satisfies all formulas in $\mathcal{H}_{2}$.

\section{Proof of Theorem 4}

To simplify notation, we will identify each set $Q$ of squares with the set of atoms $q(\bar{i}, \bar{j})$ where $(i, j) \in Q$. By $D_{n}$ we denote the set of atoms of the forms $d l(\bar{i}, \bar{j}, \overline{i-j+n})$ and $d 2(\bar{i}, \bar{j}, \overline{i+j-1})$ for all $i, j$ from $\{1, \ldots, n\}$. Recall that the rules of the program $K$ are denoted by $R_{1}, \ldots, R_{7}$.

Lemma 1

A set of atoms is a stable model of

$$
\tau R_{1} \cup \tau R_{4} \cup \tau R_{5}
$$

iff it is of the form $Q \cup D_{n}$ where $Q$ is a set of squares.

Proof

We can turn (35) into a strongly equivalent infinitary program as follows. The result of applying $\tau$ to $R_{1}$ is (21). Each conjunctive term in this formula is strongly equivalent to

$$
\neg \neg q(\bar{i}, \bar{j}) \rightarrow q(\bar{i}, \bar{j}) .
$$

The set $\tau R_{4}$ is strongly equivalent to the set of formulas

$$
\top \rightarrow d 1(\bar{i}, \bar{j}, \overline{i-j+n})
$$

$(1 \leq i, j \leq n)$. (We take into account that $\tau(\bar{i}=\overline{1} . \cdot \bar{n})$ is equivalent to $\top$ if $1 \leq i \leq n$ and to $\perp$ otherwise, and similarly for $j$.) Similarly, $\tau R_{5}$ is strongly equivalent to the set of formulas

$$
\top \rightarrow d 2(\bar{i}, \bar{j}, \overline{i+j-1})
$$

$(1 \leq i, j \leq n)$. Consequently, (35) is strongly equivalent to the conjunction $H$ of formulas (36)-(38). It is easy to check that $H$ is an extratight infinitary program, so that by Proposition 1 its stable models are identical to its supported models. A set $I$ of atoms is a model 
of $H$ iff $D_{n} \subseteq I$. Furthermore, $I$ is supported iff every element of $I$ has the form $q(\bar{i}, \bar{j})$ or is an element of $D_{n}$. Consequently, supported models of $H$ are sets of the form $Q \cup D_{n}$ where $Q$ is a set of squares.

Lemma 2

A set $I$ of atoms is a stable model of $\tau K$ iff it has the form $Q \cup D_{n}$, where $Q$ is a solution to the $n$-queens problem.

Proof

Let $\mathcal{H}_{1}$ be (35) and $\mathcal{H}_{2}$ be

$$
\tau R_{2} \cup \tau R_{3} \cup \tau R_{6} \cup \tau R_{7} .
$$

All formulas in $\mathcal{H}_{2}$ are constraints. Consequently, by Proposition 2, $I$ is a stable model of $\tau K$ iff it is a stable model of $\mathcal{H}_{1}$ and satisfies all formulas in $\mathcal{H}_{2}$. By Lemma $1, I$ is a stable model of $\mathcal{H}_{1}$ iff it is of the form $Q \cup D_{n}$, where $Q$ is a set of squares. It remains to show that a set $I$ of the form $Q \cup D_{n}$ satisfies all formulas in $\mathcal{H}_{2}$ iff $Q$ is a solution to the $n$-queens problem. Specifically, we will show that for any set $I$ of the form $Q \cup D_{n}$

(i) $I$ satisfies $\tau R_{2}$ iff for all $i \in\{1, \ldots, n\}, I$ contains exactly one atom of the form $q(\bar{i}, \bar{j})$

(ii) $I$ satisfies $\tau R_{3}$ iff for all $j \in\{1, \ldots, n\}, I$ contains exactly one atom of the form $q(\bar{i}, \bar{j})$;

(iii) $I$ satisfies $\tau R_{6} \cup \tau R_{7}$ iff no two squares in $I$ are in the same diagonal.

To prove (i), note first that $\tau R_{2}$ is equivalent to the set of formulas

$$
\neg \neg(\tau(\operatorname{count}\{Y: q(\bar{i}, Y)\}=\overline{1}))
$$

$(1 \leq i \leq n)$. By Theorem 1 , this set is strongly equivalent to the set of formulas

$$
\neg \neg(\tau(\operatorname{count}\{Y: q(\bar{i}, Y)\} \leq \overline{1}) \wedge \tau(\operatorname{count}\{Y: q(\bar{i}, Y)\} \geq \overline{1})) .
$$

By Theorem 3 and the comment at the end of Section 5.3, the result of applying $\tau$ to the first aggregate atom in (39) is strongly equivalent to

$$
\bigwedge_{\substack{\Delta \subseteq A \\|\Delta|=2}} \neg \bigwedge_{(1, r) \in \Delta} q(\bar{i}, r) .
$$

This formula can be written as

$$
\bigwedge_{\substack{\Sigma \subseteq P \\|\Sigma|=2}} \neg \bigwedge_{r \in \Sigma} q(\bar{i}, r),
$$

where $P$ is the set of precomputed terms. It is easy to see that $I$ satisfies this formula iff it contains at most one atom of the form $q(\bar{i}, r)$. On the other hand, by Theorem 2 , the result of applying $\tau$ to the second aggregate atom in (39) is strongly equivalent to

$$
\bigvee_{\substack{\Delta \subseteq A \\|\Delta|=1}} \bigwedge_{(1, r) \in \Delta} q(\bar{i}, r) .
$$

Similar reasoning shows that $I$ satisfies this formula iff it contains at least one atom of the form $q(\bar{i}, r)$. Since $I=Q \cup D_{n}, r$ in this atom is one of $\overline{1}, \ldots, \bar{n}$. 
Claim (ii) is proved in a similar way.

To prove (iii), note first that two squares $\left(\overline{i_{1}}, \overline{j_{1}}\right),\left(\overline{i_{2}}, \overline{j_{2}}\right)$ are in the same diagonal iff there exists a $k \in\{1, \ldots, 2 n-1\}$ such that

$$
d 1\left(\bar{i}_{1}, \bar{j}_{1}, \bar{k}\right), d 1\left(\bar{i}_{2}, \bar{j}_{2}, \bar{k}\right) \in D_{n}
$$

or

$$
d 2\left(\bar{i}_{1}, \bar{j}_{1}, \bar{k}\right), d 2\left(\bar{i}_{2}, \bar{j}_{2}, \bar{k}\right) \in D_{n} .
$$

We will show that a set $I$ of the form $Q \cup D_{n}$ does not satisfy $\tau R_{6}$ iff there exists a $k$ such that (40) holds for two distinct elements $q\left(\overline{i_{1}}, \overline{j_{1}}\right), q\left(\overline{i_{2}}, \overline{j_{2}}\right) \in Q$, and that it does not satisfy $\tau R_{7}$ iff there exists a $k$ such that (41) holds for such two elements. The result of applying $\tau$ to $R_{6}$ is strongly equivalent to the set of formulas

$$
\neg \tau(2 \leq \operatorname{count}\{\overline{0}, q(X, Y): q(X, Y), d 1(X, Y, \bar{k})\})
$$

$(1 \leq k \leq 2 n-1)$. Formula (42) is identical to

$$
\neg \tau(\operatorname{count}\{X, Y: q(X, Y), d 1(X, Y, \bar{k})\} \geq 2) .
$$

In view of Theorem 2 , it follows that it is strongly equivalent to

$$
\neg \bigvee_{\substack{\Delta \subseteq A \\|\Delta|=2}} \bigwedge_{(1,(r, s)) \in \Delta}(q(r, s) \wedge d 1(r, s, \bar{k}))
$$

$(1 \leq k \leq 2 n-1)$. This formula can be written as

$$
\neg \bigvee_{\substack{\Sigma \subset P \times P \\|\Sigma|=2}} \bigwedge_{(r, s) \in \Sigma}(q(r, s) \wedge d 1(r, s, \bar{k})) .
$$

For any set $Q$ of squares,

$$
Q \cup D_{n} \text { does not satisfy (43) }
$$

iff there exist two distinct pairs $\left(r_{1}, s_{1}\right),\left(r_{2}, s_{2}\right)$ from $P \times P$ such that $q\left(r_{1}, s_{1}\right), q\left(r_{2}, s_{2}\right) \in Q$ and $d 1\left(r_{1}, s_{1}, \bar{k}\right), d 1\left(r_{2}, s_{2}, \bar{k}\right) \in D_{n}$

iff there exist two distinct squares $\left(\bar{i}_{1}, \bar{j}_{1}\right),\left(\bar{i}_{2}, \bar{j}_{2}\right) \in Q$ such that (40) holds.

The claim about (41) is proved in a similar way.

Theorem 4 is immediate from the lemma.

\section{References}

FERRARIS, P. AND LIFSCHITZ, V. 2005. Mathematical foundations of answer set programming. In We Will Show Them! Essays in Honour of Dov Gabbay. King's College Publications, 615-664.

LIFSCHITZ, V. AND YANG, F. 2013. Lloyd-Topor completion and general stable models. Theory and Practice of Logic Programming 13, 4-5. 